Kompetente Antworten innerhalb von 48 Stunden!
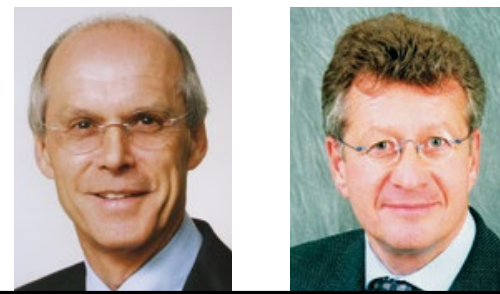

Dr. med. P. Stiefelhagen

Internist,

Hachenburg

\title{
Laborbefunde
}

\section{Wieso bleibt der ASL-Titer anhaltend erhöht?}

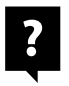
Dr. Heinrich Hümmer, Herrsching, Bayern: Ich beobachte bei meinen Patienten immer wieder über lange Zeit anhaltende, deutlich erhöhte ASLTiter, ohne irgendwelche Anhaltspunkte für eine bestehende Streptokokkeninfektion - auch nicht im Abstrich aus der Mundhöhle. Diese Patienten klagen allerdings häufiger über arthralgische Beschwerden bei unauffälliger Rheumaserologie und im Normbereich befindlichen Entzündungsparametern (CCP, ANA, AMA, RF, $B K S, C R P$, Eisen, Ferritin u. ä.).

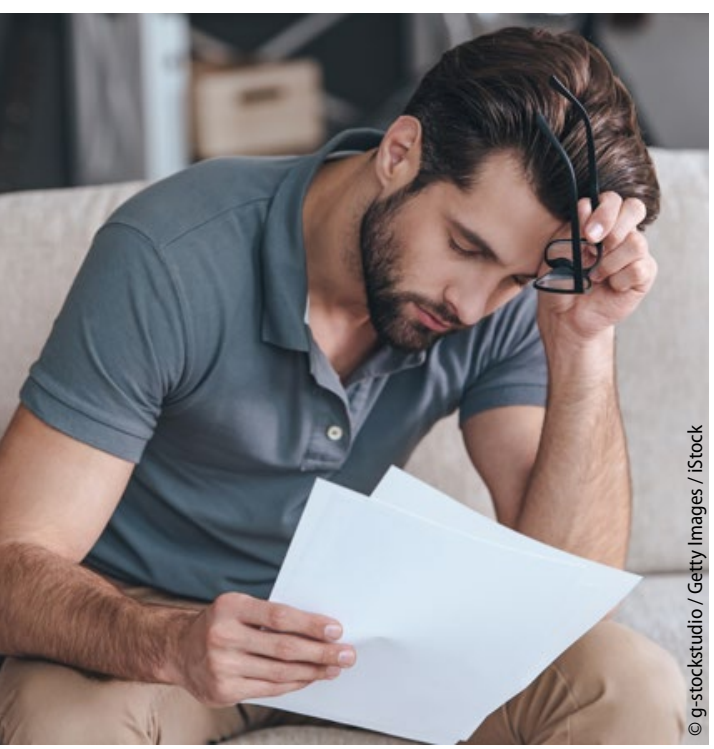

Laborbefunde können viele unbegründete Sorgen auslösen.

\section{Auf die Frage nach der Bedeutung} eines länger erhöhten ASL-Titers ohne Hinweis auf Streptokokken erhielt ich von einem angesehenen Immunologen die lapidare Antwort, diesen Wert einfach nicht mehr zu bestimmen.

Die Frage nun: Gibt es neuere Erkenntnisse zur Aussagekraft eines chronisch erhöhten ASL-Titers? Gibt es Hinweise, dass dieser eine unspezifische, chronische Immunaktivierung ggf. auch im Sinne einer beginnenden autoimmunologischen Aktivierung widerspiegeln könnte?

a

MMW-Experte Stiefelhagen: Mir sind keine neuen Erkenntnisse zu diesem Problem bekannt. Ich möchte mich der Empfehlung des Kollegen anschließen, diesen Parameter gar nicht mehr zu bestimmen, da sich daraus keinerlei weitere diagnostische und auch keine therapeutische Konsequenzen ergeben.

!

MMW-Experte FüeßI: Die ASL-Bestimmung ist ein Test zum Nachweis eines Antikörpers gegen das Bakterium Streptococcus pyogenes der Gruppe A. Antikörper gegen dieses Bakterium in unterschiedlicher Titerhöhe kommen bei vielen Menschen vor, ohne dass diese deshalb krank sind. Es handelt sich um einen sogenannten Durchseuchungstiter, populär auch „Serumnarbe“ genannt, wie ihn viele Infektionen, sei es mit oder ohne durchgemachte klinisch manifeste Krankheit hinterlassen. Eine Beurteilung von Blutwerten und die Zuordnung zu bestimmten Krankheiten sind immer nur in Zusammenhang mit den jeweiligen klinischen Beschwerden und Krankheitsmanifestationen möglich.

Diese Erkenntnis ist bei vielen Ärzten nicht in ausreichendem Maß vorhanden. Mit dem ungezielten serologischen Screening und einer falschen Interpretation von Laborbefunden werden in der Medizin leider viel Verwirrung und Unheil angerichtet und viele Patienten verunsichert. Ein typisches Beispiel ist die Borrelien-Serologie.

Die ASL-Bestimmung wird vielfach bei unklaren Gelenkbeschwerden auf der Suche nach einer „rheumatischen Erkrankung“ eingesetzt. In den Köpfen sitzt noch die in Europa mittlerweile verschwundene Erkrankung des rheumatischen Fiebers. Nachdem sowohl Gelenkbeschwerden (meist degenerativer Natur) als auch durchgemachte Streptokokkeninfektionen sehr häufig sind, muss es rein statistisch auch häufig vorkommen, dass beide Phänomene beim selben Menschen vorhanden sind, obwohl sie kausal nichts miteinander zu tun haben.

Da kann man nur sagen: Hände weg von den ungezielten „Laborlatten“, die äußerst selten zu einer Diagnose, aber sehr häufig zu interpretatorischen Folgeproblemen führen! 